\title{
Failure mechanisms of titanium VT1-0 and zirconium alloy E110 in ultrafine-grained, fine-grained and coarse-grained states under cyclic loading in gigacycle regime
}

\author{
O. B. Naimark ${ }^{1}$, Yu. P. Sharkeev ${ }^{2,3}$, A. M. Mairambekova ${ }^{4, \dagger}$, M. V. Bannikov ${ }^{1}$, \\ A. Yu. Eroshenko' ${ }^{2}$ A. I. Vedernikova ${ }^{1}$ \\ †aikol@ispms.tsc.ru \\ ${ }^{1}$ Institute of Continuous Media Mechanics UB RAS, 1 Academician Korolev str., Perm, 614013, Russia \\ ${ }^{2}$ Institute of Strength Physics and Materials Science SB RAS, 2/4 Akademicheskii ave., Tomsk, 634055, Russia \\ ${ }^{3}$ National Research Tomsk Polytechnic University, 30 Lenin ave., Tomsk, 634050, Russia \\ ${ }^{4}$ National Research Tomsk State University, 36 Lenin ave., Tomsk, 634050, Russia
}

\begin{abstract}
Fatigue tests were carried out on samples of titanium VT1-0 and zirconium alloy $\mathrm{Zr}-1 \mathrm{wt} \% \mathrm{Nb}$ in the ultrafine-grained, fine-grained and coarse-grained states in a gigacycle fatigue regime. It was found that the formation of an ultrafine-grained structure led to an increase in the fatigue limit in the gigacyclic region ( $10^{9}$ cycles) by 1.3 times for titanium and 1.7 times for zirconium alloy when compared to the fine-grained and coarse-grained states. An evolution of the temperature field for titanium and zirconium alloy samples in various structural states in the process of cyclic loading was studied by the method of infrared thermography. It was shown that the process of cyclic deformation in all types of structural states was accompanied by an initiation and expansion of a heat source in a local volume of samples which has a significant impact on the fatigue strength. The increment of the maximum temperature on the surface of ultrafine-grained samples of titanium VT1-0 and zirconium alloy $\mathrm{Zr}-1 \mathrm{wt} \% \mathrm{Nb}$ is significantly lower than that for the fine-grained and coarse-grained states. This fact indicates a qualitative change in the mechanism of energy dissipation which is associated with characteristic features of the ultrafine-grained state. When comparing the dynamics of thermal fields for the titanium and zirconium alloy samples in coarse-grained, fine-grained and ultrafine-grained states, it was found that the energy dissipation zone covered a considerable volume of the sample in the process of fatigue tests in case of ultrafine-grained state, whereas in case of coarse-grained and fine-grained states the growth of thermal energy was localized in the gauge area of the sample.
\end{abstract}

Keywords: titanium, zirconium alloy, ultrafine-grained structure, gigacycle fatigue, infrared thermography.

УДК: 539.5, 539.4

\section{Закономерности разрушения титана ВТ1-0 и сплава циркония Э110 в ультрамелкозернистом, мелкозернистом и крупнокристаллическом состояниях} при циклическом нагружении в гигацикловом режиме Наймарк О. Б. ${ }^{1}$, Шаркеев Ю. П. ${ }^{2,3}$, Майрамбекова А. М. ${ }^{4, \dagger}$, Банников М. Б. ${ }^{1}$, Ерошенко А. Ю. ${ }^{2}$, Ведерникова А.И. ${ }^{1}$

${ }^{1}$ Институт механики сплошных сред УрО РАН, ул. Академика Королева 1, Пермь, 614013, Россия ${ }^{2}$ Институт физики прочности и материаловедения СО РАН, Академический пр-т 2/4, Томск, 634055, Россия ${ }^{3}$ Национальный исследовательский Томский политехнический университет, пр-т Ленина 30, Томск, 634050, Россия ${ }^{4}$ Национальный исследовательский Томский государственный университет, пр-т Ленина 36, Томск, 634050, Россия

Проведены усталостные испытания для образцов титана ВT1-0 и сплава циркония $\mathrm{Zr}-1$ мас. \% Nb в ультрамелкозернистом, мелкозернистом и крупнокристаллическом состояниях в режиме гигацикловой усталости. Установлено, что формирование ультрамелкозернистой структуры в титане и сплаве циркония приводит к увеличению предела 
усталости титана в 1.3 раза, а сплава циркония в 1.7 раза в гигацикловой области (109 циклов) по сравнению с мелкозернистым и крупнозернистым состоянием. Методом инфракрасной термографии проведено исследование эволюции температурного поля для образцов титана и сплава циркония в различных структурных состояниях в процессе циклического нагружения. Показано, что процесс циклического деформирования для всех типов структурных состояний сопровождается зарождением и распространением очага тепловыделения в локальном объеме образцов и оказывает существенное влияние на величину усталостной прочности. Приращение максимальной температуры на поверхности ультрамелкозернистых образцов титана ВT1-0 и сплава циркония $\mathrm{Zr}-1$ мас. \% $\mathrm{Nb}$ существенно ниже, чем для мелкозернистого и крупнокристаллического состояний, что свидетельствует о качественном изменении процесса диссипации поглощения энергии, связанной с особенностями ультрамелкозернистого состояния. При сопоставлении динамики изменения тепловых полей для образцов титана и сплава циркония в крупнокристаллическом, мелкозернистом и ультрамелкозернистом состояниях, установлено, что зона диссипации энергии охватывает значительный объем образца в процессе усталостных испытаний для случая ультрамелкозернистого состояния, тогда как в случае крупнокристаллического и мелкозернистого состояния рост тепловой энергии имеет локализованный характер в рабочей зоне образца.

Ключевые слова: титан, сплав циркония, ультрамелкозернистая структура, гигацикловая усталость, инфракрасная термография.

\section{1. Введение}

Научный и практический интерес представляют титановые и циркониевые сплавы. Наиболее широко применяемыми в медицине являются «чистый» титан и титановые сплавы [1]. Циркониевые сплавы $\mathrm{Zr}-1 \mathrm{Nb}$, $\mathrm{Zr}-2.5 \mathrm{Nb}$ (промышленные сплавы марок Э110, Э125) благодаря высокой коррозионной стойкости находят применение не только в качестве конструкционных материалов в атомной энергетике, но и в медицинских приложениях [2]. Сдерживающим фактором для широкого практического применения «чистого» титана и сплавов циркония является невысокий уровень механических свойств, в том числе, усталостная прочность и циклическая долговечность.

Создание наноструктурной (НС) и/или ультрамелкозернистой (УМЗ) структуры в металлах и сплавах методами интенсивной пластической деформации (ИПД) позволяет кардинально повысить механические свойств материалов (пределы текучести и прочности, твердость, предел выносливости и циклическая долговечность и др.) [3]. Это обусловлено тем, что металлы в НС и УМЗ состоянии имеют ряд особенностей как малый размер зерна, наличие преимущественно большеугловых границ зерен и существование высоких внутренних напряжений, вызванных большой плотностью дефектов. Формирование НС/УМЗ состояния в металлах эффективно повышает предел выносливости в области многоцикловой усталости (более $10^{6}$ циклов) [4,5]. В настоящее время актуальными являются исследования по влиянию НС и/или УМЗ состояния на процессы разрушения при циклических нагрузках, анализу кривой усталости в широком диапазоне количества циклов нагружения, не только для многоцикловой усталости, но и для гигацикловой усталости, т. е. более $10^{9}$ циклов [6,7].

Особенности диссипации энергии, сопровождающей эволюцию микроструктуры при циклическом деформировании, необходимо учитывать при анализе механизмов зарождения и распространения усталостных трещин в местах локализации пластической деформации. В свою очередь, процессы локализации деформации сопровождаются интенсивным выделением тепла [8]. Поэтому перспективным является применение метода инфракрасной термографии, который позволяет исследовать закономерности преобразования и накопления энергии в процессе деформирования и получать информацию об изменении микроструктуры и накоплении структурных дефектов на ранних этапах разрушения [8-10]. Вопросы, связанные с исследованиями закономерностей преобразования и накопления энергии в процессе деформирования при циклическом нагружении, остаются до настоящего времени недостаточно изученными.

Целью настоящей работы является установление закономерностей деформации и разрушения титана ВТ1-0 и сплава циркония Э110 в УМЗ состоянии при циклическом нагружении в режиме гигацикловой усталости.

\section{2. Материалы и методы исследования}

В качестве объектов исследования были выбраны «технически чистый» титан марки ВТ1-0 и сплав Э110 состава $\mathrm{Zr}-1$ мас. \% Nb (Zr-1Nb). УМЗ структуру в исследуемых материалах формировали комбинированным двухэтапным методом ИПД, который включал многократное abc-прессование и многоходовую прокатку в ручьевых валках с последующим отжигом $[11,12]$. Микроструктуру и фазовый состав исследовали с помощью оптической микроскопии (микроскоп Carl Zeiss Axio Observer), просвечивающей электронной микроскопии (микроскоп JEOL JEM 2100). Средний размер элементов структуры (зерна, субзерна, фрагменты) рассчитывали методом секущей [13].

Испытания на гигацикловую усталость проводили на ультразвуковой резонансной нагружающей машине Shimadzu USF-2000 при заданных амплитудах напряжений 100-300 МПа, частоте циклических колебаний 20 кГц с коэффициентом асимметрии цикла $R=-1$. Преимуществом данной машины является возможность достигать количества циклов нагружения $10^{9}$ и более за достаточно короткое время по сравнению с классической испытательной машиной с частотой испытаний в $50-100$ Гц $[6,7,9]$.

Все исследования были выполнены для титана и сплава циркония в УМЗ состоянии, а также для крупнокристаллического (КК) титана и мелкозернистого 
(M3) сплава циркония. КК состояние в титане и МЗ состояние в сплаве циркония получали путем рекристаллизационных отжигов в течение одного часа в вакууме УМЗ образцов: для титана - при $800^{\circ} \mathrm{C}$, для сплава циркония - при $580^{\circ} \mathrm{C}$. Из заготовок титана BT1-0 и сплава циркония в УМЗ, КК и МЗ состояниях были изготовлены образцы для усталостных испытаний в виде цилиндрических лопаток. Геометрические размеры лопаток определяли по аналитическим формулам [6] в зависимости от плотности исследуемого материала и его динамического модуля Юнга, отвечающим условиям стоячей ультразвуковой волны и резонанса испытательной системы, чтобы максимум стоячей волны напряжения приходился на центр образца, а максимумы волны смещения на его торцы. Для предотвращения выхода системы из резонанса за счет изменения его упругих свойств в результате нагрева образцов в ходе усталостных испытаний до температур $\left(200-400^{\circ} \mathrm{C}\right.$ и более) использовали охлаждение потоками воздуха.

Для регистрации температурного поля в ходе циклического деформирования образцов использовали инфракрасную камеру FLIR SC 5000. Эволюция температурного поля в процессе циклического деформирования записывалась в виде цифровых термограмм, полученных с помощью измерительной тепловизионной системы в режиме реального времени. Запись поля температуры поверхности образца проводилась с частотой 2234.6 Гц и минимальным пространственным разрешением $2 \cdot 10^{-4}$ м.

Для калибровки инфракрасной камеры использовалась стандартная калибровочная таблица. Инфракрасная камера позволяла фиксировать температуру до $300{ }^{\circ} \mathrm{C}$. Анализ и обработку результатов, визуализацию температурных полей в виде графических и иллюстративных данных осуществляли с помощью специализированной программы Altair. С целью улучшения качества визуализации температурных полей предварительно поверхность лопаток покрывалась тонким слоем аморфного углерода.

\section{3. Результаты и обсуждение}

Ha Рис. S1a-d (дополнительный материал) представлена микроструктура титана и сплава циркония, сформированная комбинированным методом ИПД. Для обоих материалов характер УМЗ микроструктуры, сформированной в результате ИПД, подобный. Наблюдаются как фрагменты и субзерна, так уже и сформировавшиеся зерна. Границы зерен, субзерен и фрагментов неравновесные. Характерной особенностью микроструктуры является присутствие большого количества контуров экстинкции, свидетельствующие о наличии высоких остаточных упругих напряжений (Рис. S1a,c, дополнительный материал). В зернах, субзернах и фрагментах локализована дислокационная субструктура с наибольшей плотностью дислокаций. Средний размер структурных элементов (зерен, субзерн и фрагментов) составил $0.2 \pm 0.1$ мкм для титана и $0.22 \pm 0.1$ мкм для сплава циркония, что соответствует УМЗ состоянию.
На микродифракциях как для титана, так и для сплава циркония (Рис. S1a,c, вклейки, дополнительный материал) наблюдается большое количество точечных рефлексов разной интенсивности, расположенных по окружности, что свидетельствует о разориентировках между границами зерен и указывает на присутствие как большеугловых, так и малоугловых разориентаций на границах субзерен и зерен. На микродифракциях для сплава циркония (Рис. S1c, вклейки, дополнительный материал) присутствуют рефлексы высокой интенсивности от основной фазы $\alpha-\mathrm{Zr}$ (ГПУ-решетка) и низкой интенсивности от фазы $\beta-\mathrm{Nb}$ (ОЦК-решетка). На светлопольных изображениях выделения ниобия явно не наблюдаются. Дополнительно частицы фазы $\beta-\mathrm{Nb}$ идентифицировали в ходе микрорентгеноспектрального анализа элементного состава. В состоянии после рекристаллизационного отжига микроструктура титана состояла из достаточно равноосных зерен со средним размером $27 \pm 8$ мкм (Рис. S2a, дополнительный материал). Сплав циркония в рекристаллизованном МЗ состоянии имел структуру с зернами $\alpha-\mathrm{Zr}$ со средним размером зерен $1.9 \pm 0.4$ мкм и расположенными по границам и в теле матричных зерен частицами ниобия со средним размером 0.4 мкм (Рис. S2c, d, дополнительный материал).

На Рис. 1 приведены экспериментальные точки и кривые усталости для титана и сплава циркония для выбранных структурных состояний. Стрелками отмечены образцы, которые не разрушились в ходе испытаний.

При амплитуде циклических напряжений $175 \mathrm{MПа}$ образцы титана в КК состоянии разрушились после $10^{5}$ циклов. Переход от КК структуры к УМЗ структуре значительно повышает выносливость титана в области

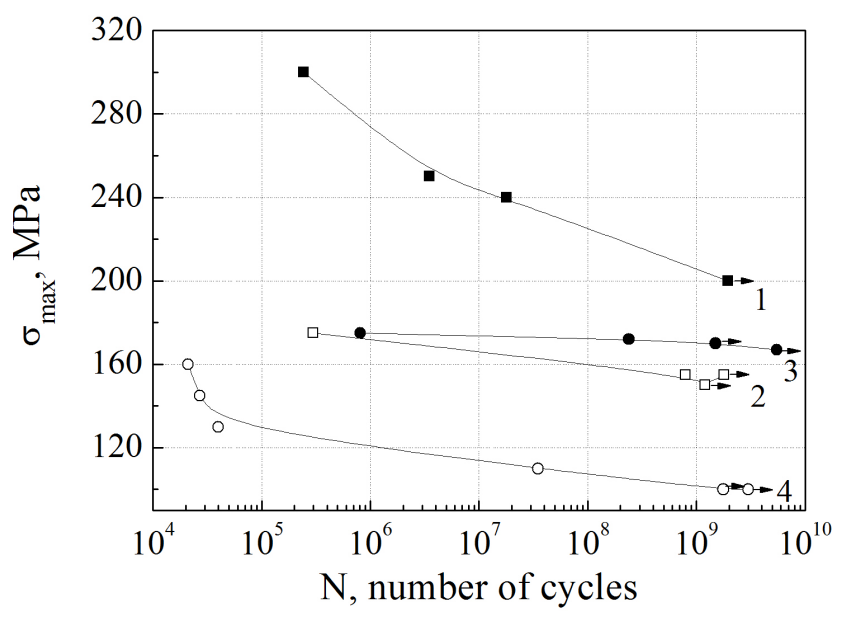

Рис. 1. Зависимость числа циклов до разрушения $N$ от амплитуды циклических напряжений $\sigma_{\max }$ для образцов в различных состояниях: - - титан в УМЗ состоянии, кривая 1; 口 - титан в КК состоянии, кривая 2; - сплав циркония в УМЗ состоянии, кривая 3; $0-$ сплав циркония в М3 состоянии, кривая 4

Fig. 1. The dependence of the number of cycles to failure $N$ on the amplitude of cyclic stresses $\sigma_{\max }$ for samples in the different states: - titanium in the UFG state (curve 1); $\square$ - titanium in the CG state (curve 2); $\bullet$ - zirconium alloy in the UFG state (curve 3); $\circ-$ zirconium alloy in the FG state (curve 4). 
гигацикловой усталости. Так при амплитуде напряжения 200 МПа число циклов нагружения до разрушения возрастает до $1.9 \times 10^{9}$. По результатам усталостных испытаний предел выносливости КК титана на базе $10^{9}$ циклов нагружения составил 155 МПа, а для УМЗ титана 200 МПа, что выше в 1.3 раза. Усталостное разрушение сплава циркония в М3 состоянии происходит в малоцикловой области $\left(N \leq 2.1 \times 10^{4}\right.$ цикла) при амплитуде напряжения $\sigma=160$ МПа. Формирование М3 состояния в сплаве циркония, приводит к повышению усталостной прочности в гигацикловом режиме нагружения. У образцов УМЗ сплава циркония число циклов нагружения до разрушения составляет $1.5 \times 10^{9}$ при напряжении 170 МПа. Для УМЗ сплава циркония предел выносливости на базе $10^{9}$ составил $100 \mathrm{MПа,} \mathrm{а} \mathrm{для} \mathrm{МЗ} \mathrm{состояния}$ в 1.7 раза выше - 160 МПа.

Исследование эволюции температурного поля образцов титана и сплава циркония при циклическом нагружении проводили методом инфракрасной термографии. Для ускорения процесса разрушения в режиме реального времени деформирование образцов проводили без принудительного охлаждения. В этом случае образцы титана и сплава циркония в различных структурных состояниях испытывали в режиме малоцикловой усталости ( $N \leq 10^{5}$ циклов нагружения) при амплитуде напряжений 130 и 100 МПа соответственно. При выбранных режимах циклического нагружения образцы титана и сплава циркония в КК были разрушены, в отличие от образцов в УМЗ состоянии, где эксперимент прерывали, когда наблюдалась стабилизация температуры в диапазоне $120-130^{\circ} \mathrm{C}$.

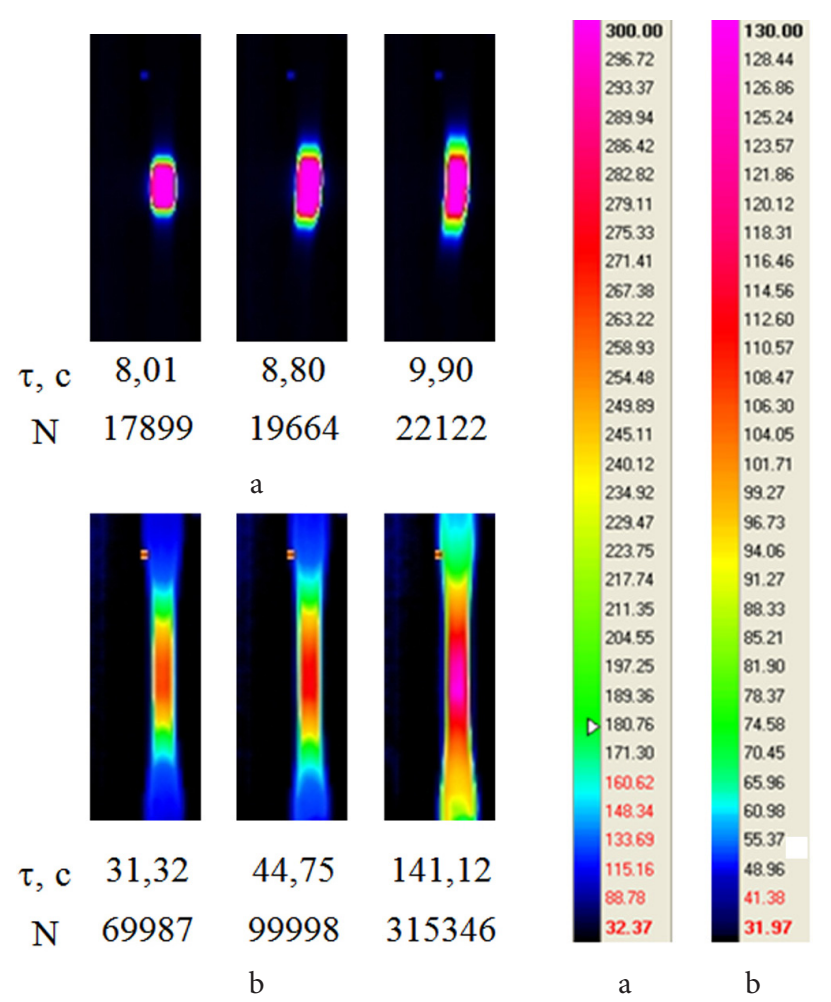

На Рис. 2 представлена эволюция теплового поля в процессе циклического нагружения образцов титана и сплава циркония в исследованных структурных состояниях ( $\tau-$ период испытания, $N-$ номер кадра к указанному моменту времени). Полученные изображения тепловых полей визуализировали изменение температуры на поверхности рабочей части образцов в режиме реального времени. Для всех типов структурных состояний исследуемых материалов наблюдается общая тенденция. Процесс циклического деформирования сопровождается зарождением и распространением очага тепловыделения, площадь которого возрастает с увеличением времени испытания.

Скорость нарастания температуры на поверхности образца титана в КК состоянии значительно выше по сравнению с УМЗ состоянием. В КК титане зона с повышенной температурой появляется в средней рабочей части образца. Затем в процессе испытаний площадь такой зоны увеличивается в размерах и распространяется по всему образцу (Рис. 2а). В случае УМ3 титана зона более высокой температуры также возникает в средней рабочей части, но в процессе испытаний ее размер увеличивается незначительно и практически не распространяется по поверхности образца (Рис. 2b).

Для сплава циркония в УМЗ состоянии распределение температуры подобно тепловому полю для титана (Рис. 2d). А для МЗ состояния зона с высокой температурой в процессе циклического нагружения занимает практически весь объем образца (Рис. 2c).

При сопоставлении динамики изменения тепловых полей для трех состояний образцов титана и сплава
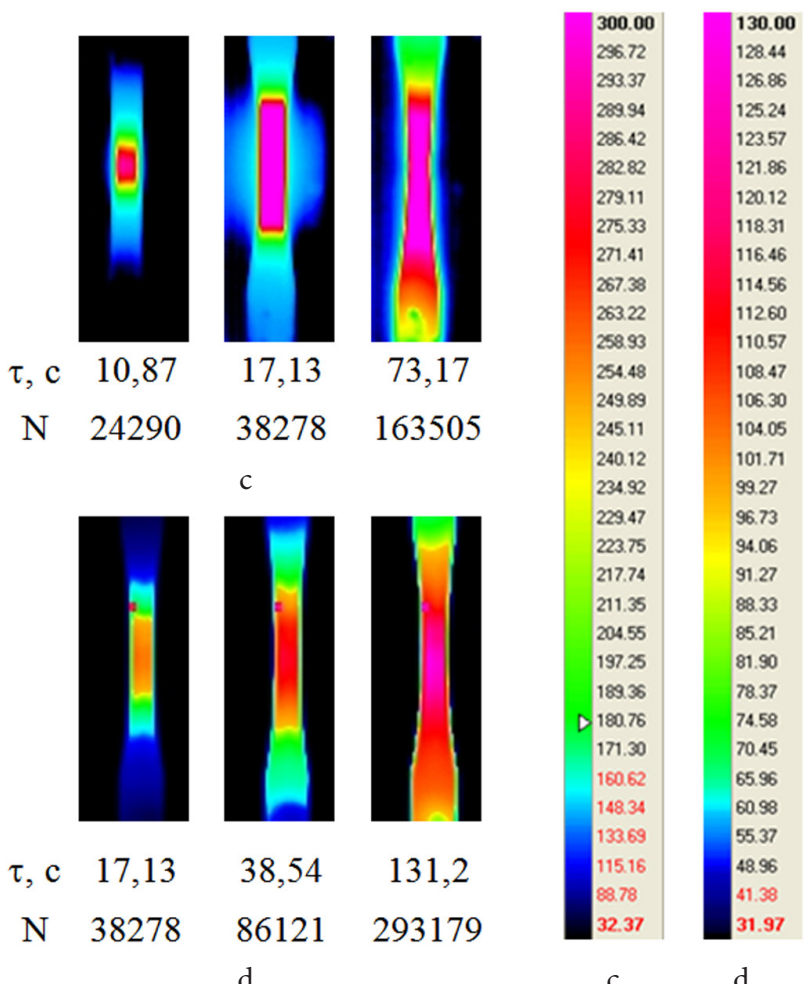

Pис. 2.(Color online) Изображения тепловых полей инфракрасной камеры в области, зарегистрированные в процессе циклического нагружения образцов титана $(\mathrm{a}, \mathrm{b})$ и сплава циркония $(\mathrm{c}, \mathrm{d})$ в различных состояниях: КК и МЗ (a,c); УМЗ состояния (b,d).

Fig. 2. (Color online) The images of the infrared camera thermal fields in the region recorded during the cyclic loading of the titanium (a,b) and zirconium alloy $(\mathrm{c}, \mathrm{d})$ samples in the different states: CG and FG (a,c); UFG states (b,d). 
циркония для КК, МЗ и УМЗ состояний видно, что локализация теплового поля наиболее выражена для КК состояния в отличие от УМЗ состояния, а для МЗ занимает промежуточное положение между УМЗ и КК состояниями. Это может быть связано с размером зерна. В случае УМЗ состояния средний размер зерна минимальный, и локализация теплового поля в средней рабочей части практически отсутствует, а для КК состояния с максимальным средним размером зерна локализация теплового поля максимально выражена. М3 состояние для сплава циркония является промежуточным и по размеру зерна, и по локализации теплового поля.

На Рис. 3 представлены зависимости максимальной температуры на поверхности образцов от времени циклического нагружения для титана и сплава циркония. Изменение максимальной температуры на поверхности фиксировалось в средней части образцов. Образцы титана в КК состоянии нагреваются до максимальной температуры $300^{\circ} \mathrm{C}$, которая достигается за 9.9 с. При этом разрушение образцов происходит при температуре выше $300^{\circ} \mathrm{C}$. Прирост температуры образцов для УМЗ титана в процессе циклического деформирования значительно меньше, чем для КК титана (Рис. За). При испытаниях образцов титана в УМЗ состоянии максимальная температура $120^{\circ} \mathrm{C}$ достигается за $141 \mathrm{c}$, что в 14 раз больше, чем для КК состояния (7.03 c). Характер изменения максимальной температуры от времени в процессе циклического нагружения образцов сплава циркония аналогичен (Рис. 3b). Разрушение образцов М3 сплава циркония, как и КК титана, происходит при температуре выше $300^{\circ} \mathrm{C}$. Данная температура достигается за 73.17 с. Появление температурного «плато» на кривых для КК титана и МЗ сплава циркония отражает тот факт, что в ходе циклического нагружения образцы были разрушены при температурах, значительно превышающих рабочий диапазон инфракрасной камеры. Температура образцов сплава циркония в УМЗ состоянии в процессе цикличе- ского деформирования достигает значения $130^{\circ} \mathrm{C}$ за время, равное 131.2 с, а в М3 состоянии за - 8.2 с.

Можно предположить, что механическая энергия в случае нагружения образцов с исходной КК структурой в большей степени «закачивается» в упругую энергию решетки в областях локализации деформации. Образцы в УМЗ состоянии обнаруживают меньшую способность к локализации деформации и задействуют большее количество «мод деформации», что приводит к уменьшению диссипативного вклада $[7,9,14]$. Приращение температуры в УМЗ образцах существенно ниже, чем для КК и МЗ образцов, т.е. циклическое нагружение титана и сплава циркония в УМЗ состоянии, сопровождается качественным изменением процесса диссипации энергии. Различия в механизмах диссипации при разрушении КК и УМЗ материалов обусловлены качественными изменениями в типах коллективных мод деформации, обусловленных дислокационными субструктурами $[10,14,15]$. Для крупнокристаллических материалов это проявляется наличием дальнодействующих полей напряжений, формированием ограниченного количества локализованных ориентационно выраженных мод пластической деформации и обусловленных ими каналов диссипации. Деформирование УМЗ материалов определяется уменьшением интенсивности дальнодействующих полей напряжений индуцированных зернограничными дефектами, и увеличением количества «мод пластической деформации». Качественные изменения в деформационных реакциях материалов при переходе от КК к УМЗ (например, нарушение закона Холла-Петча) интерпретируется в $[10,14]$ как специальный класс критических явлений, обусловленный дефектами (структурно-скейлинговые переходы). Симметрийным признаком данных переходов является резкое увеличение количества мод деформации, увеличение вклада «запасенной» энергии в полях структурных напряжений и, как следствие, уменьшение диссипативного

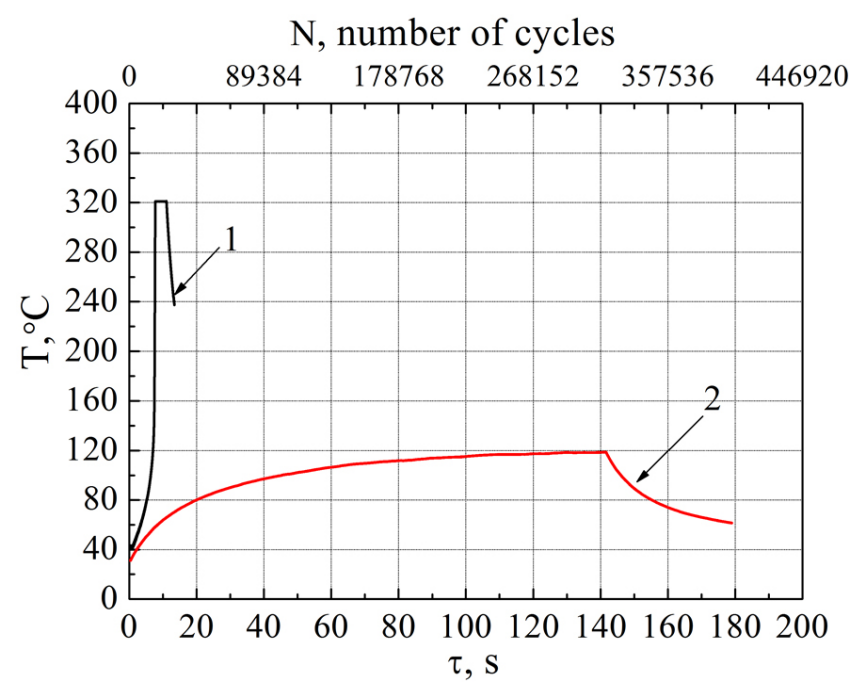

a

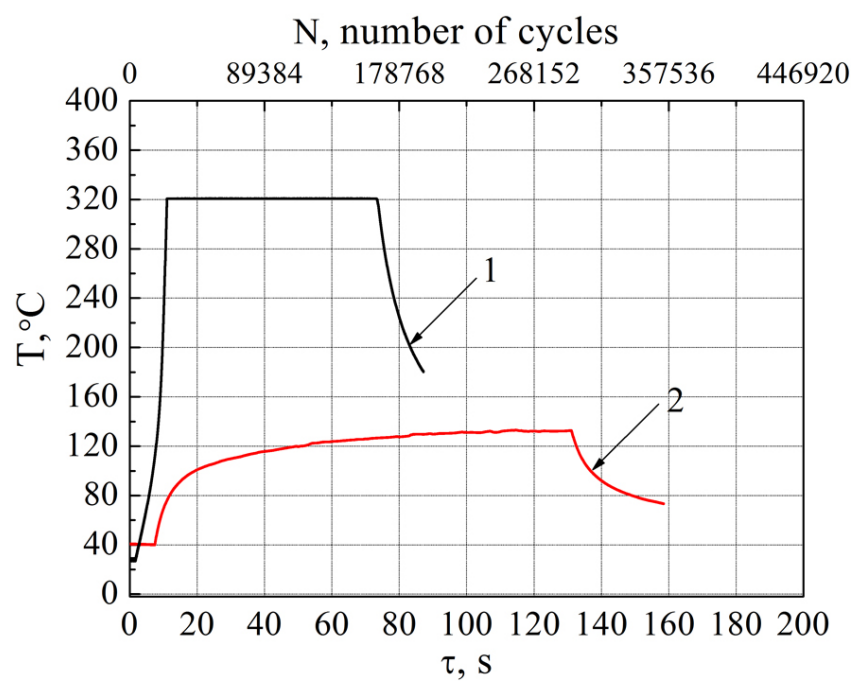

b

Рис. 3. Изменение температуры в процессе циклического нагружения титана (а) и сплава циркония (b) в структурных состояниях: 1 - КК, МЗ состояния; 2 - УМЗ состояние.

Fig. 3. The change in temperature during the cyclic loading of the titanium (a) and zirconium alloy (b) in the structural states: 1 - CG and FG states; 2 - UFG states. 
вклада. В процессе усталостного разрушения металлов большую роль играют также очаговое тепловыделение в микрообъемах, подвергающихся микродеформациям. В результате такого локального повышения температуры прочность материала в микрообъемах может уменьшаться, что облегчает формирование новых пластических сдвигов, которые в свою очередь могут способствовать дальнейшему повышению температуры. При этом в процессе деформирования в локальных полосах скольжения, на которых происходит рассеивание энергии, и температура может быть значительно выше, чем в образце в целом.

\section{4. Заключение}

Установлено повышение усталостной прочности при гигацикловых режимах нагружения для титана и сплава циркония с УМЗ структурой. Формирование УМЗ структуры приводит к увеличению предела усталости титана ВТ1-0 в 1.3 раза, а сплава циркония $\mathrm{Zr}-1$ мас.\% $\mathrm{Nb}$ в 1.7 раза в области гигацикловой усталости.

В процессе циклического нагружения приращение температуры на поверхности образцов титана и сплава циркония в УМЗ состоянии существенно ниже, чем для КК и МЗ состояний. Циклическое нагружение титана и сплава циркония в УМЗ состоянии, сопровождается качественным изменением процесса диссипации поглощения энергии.

Процесс циклического деформирования образцов титана и циркония в УМЗ, МЗ и КК состояниях сопровождается зарождением и распространением очага тепловыделения. Зона диссипации энергии охватывает значительный объем образца в процессе усталостных испытаний для случая УМЗ состояния, тогда как в случае КК и МЗ состояния рост тепловой энергии имеет локализованный характер в рабочей зоне образца.

Благодарности/Acknowledgements. Исследование выполнено при финансовой поддержке РФФИ в рамках научного проекта № 17-32-50163 «мол_нр»./The research was performed by support of Russian Foundation for Basic Research in the framework scientific project № 17-32-50163 «mol_nr».

Дополнительньйматериал/SupplementaryMaterial. Электронная версия статьи содержит дополнительньй материал (рисунки), доступньй безвозмездно на сайте журнала (www.lettersonmaterials.com)./The online version of this paper contains supplementary material (figures) available free of charge at the journal's Web site (www.lettersonmaterials.com).

\section{Литература/References}

1. L.D. Zardiackas, M.J. Kraay, H.L. Freese. Titanium, niobium, zirconium and tantalum for medical and surgical applications. ASTM International (2006) 265 p.

2. D. L. Douglass. The metallurgy of Zirconium. International Atomic Energy Agency, Vienna (1971) 160 p.

3. R.Z. Valiev, A.P. Zhilyaev, T.G. Langdon. Bulk nanostructured materials: fundamentals and applications. New Jersey, John Wiley \& Sons (2014) 456 p.

4. A.A. Shanyavsky. Phys.mesomech. 17(6), 87 (2014). DOI: 10.1134/S1029959915020095

5. I.P. Semenova, R.Z. Valiev, E. B. Yakushina, G. H. Salimgareeva, T.C. Lowe. J. Mater. Sci. 43, 7354 (2008).

6. C. Bathias, P.C. Paris. Gigacycle Fatigue in Mechanical Practice. Dekker Publisher Co, Marcel (2005) 328 p.

7. V.A. Oborin, M. V. Bannikov, O. B. Naimark, T. PalinLuc. Tech. Phys. Lett. 36(11), 1061 (2010).

8. E. A. Moyseychik, V. P. Vavilov, M. V. Kuimova. J. Nondestr. Eval. 37(2), 28 (2018). DOI: 10.1007/s10921-018-0482-4

9. O. Plekhov, O. Naimark, R. Valiev, I. Semenova, N. Saintier, T. Palin-Luc. Tech. Phys. Lett. 34(7), 557 (2008). DOI: 10.1134/S1063785008070067

10. O. B. Naimark. The Phisics of Metals and Metallography. 84, 327 (1997).

11. Yu.P. Sharkeev, A.Yu. Eroshenko, V.I. Danilov, A. I. Tolmachev, P. V. Uvarkin, Yu. A. Abzaev. Russ. Phys. J. 56, 1156 (2014). DOI: 10.1007/s11182-014-0156-3

12. A.Yu. Eroshenko, A.M. Mairambekova, Yu.P. Sharkeev, Zh. G. Kovalevskaya, M.A. Khimich, P. V. Uvarkin. Letters on Materials. 7(4), 469 (2017). DOI: 10.22226/2410-3535-2017-4-469-472

13. ASTM E1382-97 (2010) Standard Test Methods for Determining Average Grain Size Using Semiautomatic and Automatic Image Analysis.

14. O. B. Naimark, Yu. V. Bayandin, V.A. Leontiev. Phys. Mesomech. 12(5-6), $239 \quad$ (2009). DOI: 10.1016/j.physme.2009.12.005

15. V.I. Betekhtin, A.G. Kadomtsev, M.A. Narykova, M. V. Bannikov, S. G. Abaimov, I.S. Akhatov, T. PalinLuc, O. B. Naimark. Phys. Mesomech. 20, 78 (2017). DOI: $10.1134 /$ S1029959917010076 